\title{
CONVERSION OF TOTAL EXTRAPERITONEAL (TEP) TO TRANSABDOMINAL PREPERITONEAL (TAPP) FOR REPAIR OF INGUINAL HERNIA; FREQUENCY AND CAUSES.
}

1. MBBS, FCPS

Assistant Professor General Surgery Hamdard College of Medicine and Dentistry.

2. MBBS, FCPS (General Surgery) Assistant Professor General Surgery Sir Syed College for Medical Sciences for Girls.

3. MBBS, FCPS, FCIS

Professor and Head Neurosurgery Bahria International Hospital Karachi.

4. MBBS, FCPS (General Surgery) Senior Registrar

Dow University of Health Sciences, Karachi.

5. MBBS

House Officer Surgery

Dow University of Health Sciences Karachi.

Correspondence Address:

Dr. Syed M. Sheraz Raza Naqvi

Dow University of Health Sciences

Karachi.

drsherazrazanaqvi@gmail.com

Article received on:

19/01/2019

Accepted for publication:

06/05/2019
Muhammad Ghayasuddin', Fareya Usmani², Imran Munir ${ }^{3}$, Mahwash Anjum Shafiq ${ }^{4}$, Syed Muhammad Sheraz Raza Naqvi ${ }^{5}$

ABSTRACT... Objectives: Our study aims to determine the incidence of and reasons for the conversion of hernia operations from laparoscopic guided total extraperitoneal (TEP) to transabdominal preperitoneal inguinal hernia repair (TEPP). Study Design: Cross-sectional study. Setting: KVSS Site Hospital Karachi, Pakistan. Period: From August 2016 to November 2018. Material \& Methods: The sampling technique utilized was consecutive non-probability sampling. The study population consisted of patients between the ages of 22 and 50 years, males, having unilateral inguinal hernia, both direct and indirect presentation. A complete history and physical examination was performed and recorded in a pre-designed proforma for all the patients. Laboratory investigations were performed per routine. The patients underwent laparoscopic total extraperitoneal hernia repair and were noted for complications and conversion to transabdominal preperitoneal inguinal hernia repair. Data was analyzed using IBM SPSS statistics version 20. Chi-square test was used to analyze categorical variables, while mean and percentages were used for numerical data. A p value of less than 0.05 is considered to be statistically significant. Results: The study population was $n=150$, mean age of the patients was $36.25+/-7.08$ with a minimum of 22 and a maximum of 50 years. The direct hernia was present in $47.33 \%$ (71 patients) while an indirect hernia was present in $52.67 \%$ (79 patients). Out of the total patient population, 21 (14\%) of the patients (having a p-value of 0.013 ) were converted to the TAPP procedure of which $10(14.08 \%)$ has a direct hernia and $11(13.92 \%)$ had an indirect hernia respectively (having a p-value of 0.953 ). The most common reason for conversion was peritoneal tear, hemorrhage and instrument failure. Conclusion: According to the results of our study, $14 \%$ of cases of direct and indirect hernia had to convert from TEP to TAPP procedure, and the main reasons for this conversion were found to be hemorrhage, peritoneal tear and instrument failure.

Key words: Hernia Repair, Inguinal Hernia, TEP, TEPP, Total Extra Peritoneal, Trans Abdominal Preperitoneal Inguinal Hernia Repair.

Article Citation: Ghayasuddin M, Usmani F, Munir I, Shafiq MA, Naqvi SMSR. Conversion of Total Extraperitoneal (TEP) to Transabdominal Preperitoneal (TAPP) for repair of inguinal hernia; Frequency and causes. Professional Med J 2020; 27(2):242-245. DOI: 10.29309/TPMJ/2020.27.2.3157

\section{INTRODUCTION}

Symptoms of an inguinal hernia can be found in $66 \%$ of the patients, however, in the rest of the population, it is symptomless. ${ }^{1}$

These symptoms include discomfort and pain of varying degrees exacerbated by certain activities such as exercise, coughing and constipation among others. The most common type of spontaneous herniae is the inguinal hernia, occurring in about $90 \%$ of the patient population. ${ }^{2}$

Hernia can be managed conservatively, or through surgery. Pain and strangulation are the major factors that prompt the surgeon to perform the repair operation. ${ }^{3}$

There are two methods for repair of hernia namely the open tension-free repair by Lichtenstein repair and the second one being a laparoscopic procedure. $^{4}$

The laparoscopic procedure is used in the treatment of recurring hernias as there is minimal scar tissue formation after the procedure which facilitates dissection and placement of the 
mesh. The most commonly used techniques for laparoscopic repair are the total extra peritoneal (TEP) and trans abdominal preperitoneal inguinal hernia repair (TAPP). ${ }^{5,6}$

Many surgeons prefer the total extraperitoneal technique (TEP) as the results are better, however, the procedure requires precision and careful technique as the working space is narrow and the pelvic anatomy might be unfamiliar to some surgeons. ${ }^{7,8,9,10}$

For patients who have undergone prior abdominal surgeries such as radical prostatectomy or cesarian section, the TEP procedure becomes difficult for the surgeons to perform. In this study, we have focused on the conversion of total extra peritoneal repair to trans abdominal preperitoneal inguinal hernia repair discussing the various aspects surrounding this change of procedure, which is prevalent in $11 \%$ of the TEP procedures. ${ }^{11,12}$

We hope to enlighten the surgical community around this conversion of procedure and hopefully provide a few recommendations as well.

\section{MATERIAL \& METHODS}

The type of study is a cross-sectional study conducted at a large tertiary care hospital in Karachi Pakistan from August 2016 to November 2018. The ethics approval for the study was provided by the ethics committee. The sampling technique utilized was consecutive nonprobability sampling. The study population consisted of patients between the ages of 22 and 50 years, males, having unilateral inguinal hernia, both direct and indirect presentation. The patients who were excluded were those who were obese a BMI of greater than $30 \mathrm{~kg} / \mathrm{m}^{2}$, previous lower abdominal surgical procedures, transurethral electrovaporization of the prostate (TVP) and appendectomy, patients with comorbidities, terminally ill and chronic conditions such as known cardiovascular, respiratory and psychiatric diseases. All the patients signed informed consent to participate in the study in a language they could comprehend.
A complete history and physical examination was performed and recorded in a pre-designed proforma for all the patients. Laboratory investigations were performed per routine such as complete blood count, lipid profile, liver function tests, urea, creatinine and electrolytes among others. Specific examinations and investigations for the surgical procedure were performed using a standard technique. ${ }^{13}$

The patients underwent laparoscopic total extraperitoneal hernia repair and were noted for complications and conversion to transabdominal preperitoneal inguinal hernia repair. Data were analyzed using IBM SPSS statistics version 20 . Chi-square test was used to analyze categorical variables, while mean and percentages were used for numerical data. A p value of less than 0.05 is considered to be statistically significant.

\section{RESULTS}

The study population was $n=150$ (all male patients), the mean age of the patients was 36.25 +/- 7.5 with a minimum of 22 and a maximum of 50 years. The majority of the patients were residents of urban areas that is 90 (60\%) while the rest were from surrounding rural 60 (40\%), $72(48 \%)$ were smokers while the rest were nonsmokers. 48 (32\%) were hypertensive while the rest were normotensive. Direct hernia was present in $47.33 \%$ (71 patients) while indirect hernia was present in $52.67 \%$ (79 patients). The patient demographics according to age group is $41(27.33 \%)$ patients in between 22 and 30 years of age, $70(46.66 \%)$ of patients between 31 and 40 years of age and 39(26\%) of patients between 41 and 50 years of age respectively.

Out of the total patient population, 21 (14\%) of the patients (having a p-value of 0.013 ) were converted to the TAPP procedure of which 10 (14.08\%) has direct hernia, and 11 (13.92\%) had indirect hernia respectively (having a p-value of 0.953). The most common reason for conversion was peritoneal tear, other variables can be found in Table-l. 


\begin{tabular}{|c|c|c|}
\hline \multirow[t]{3}{*}{ Variable } & & \\
\hline & \multicolumn{2}{|c|}{ Conversion to TAPP } \\
\hline & Yes & No \\
\hline \multicolumn{3}{|l|}{ Age Group } \\
\hline 22 to 30 years & $6(14.6 \%)$ & $36(87.80 \%)$ \\
\hline 31 to 40 years & $5(7.14 \%)$ & $66(94.28 \%)$ \\
\hline 41 to 50 years & $10(25.64 \%)$ & $30(76.92 \%)$ \\
\hline \multicolumn{3}{|l|}{ Type of Hernia } \\
\hline Direct hernia & $10(14.08 \%)$ & $61(85.91 \%)$ \\
\hline \multirow[t]{3}{*}{ Indirect hernia } & $11(13.92 \%)$ & 69 (87.34\%) \\
\hline & $\begin{array}{l}\text { Type of } \\
\text { Hernia }\end{array}$ & \\
\hline & Direct & Indirect \\
\hline \multicolumn{3}{|c|}{ Reason for Conversion } \\
\hline \multicolumn{3}{|l|}{ Hemorrhage } \\
\hline Yes & $4(5.63 \%)$ & $4(5.06 \%)$ \\
\hline No & $67(94.36 \%)$ & 76 (96.20\%) \\
\hline \multicolumn{3}{|l|}{ Peritoneal Tear } \\
\hline Yes & $5((7.04 \%)$ & $6(7.59 \%)$ \\
\hline No & $66(83.54 \%)$ & $74(93.67 \%)$ \\
\hline \multicolumn{3}{|c|}{ Instrument Failure } \\
\hline Yes & $1(1.4 \%)$ & $1(1.26 \%)$ \\
\hline No & 70 (98.59\%) & $78(98.73 \%)$ \\
\hline
\end{tabular}

\section{DISCUSSION}

In our study all the patients studied were male having a mean age of $36.25+/-7.5$ years and age range of 28 years with a lower limit of 22 and an upper limit of 50 years respectively. The type of hernia was a direct hernia in $71(47.33 \%)$ patients and an indirect hernia in 79 (52.66\%) of the patients. In a similar study by Dulucq et al indirect hernias were more common than a direct hernia. ${ }^{14,15}$ In a study by Ates et al they found the frequency of conversion of the procedure to be $11 \%$ while in our study the conversion rate was found to be $14 \%$, the reasons for the conversion were hemorrhage secondary to injury to the inferior epigastric vessel, which causes obstruction to the field of vision. Peritoneal tear and instrumental failure were also the major reasons for conversion to TAPP procedure as surgeons were not able to perform the procedure in a narrow environment and the instruments were not able to function properly. When it comes to reasons for conversion to TAPP procedure hemorrhage $8(5.33 \%)$ was the major culprit, it was present in $4(5.06 \%)$ of patients indirect hernia and $4(5.6 \%)$ of patients with direct hernia, the $\mathrm{p}$-value of this conversion was found to be 0.862 . Peritoneal tear was found in $11(7.33 \%)$ of patients among whom 5(7.04\%) had direct hernia and $6(7.59 \%)$ had indirect hernia respectively having a p-value of 0.9140 . Instrument failure was present in $2(1.33 \%)$ of patients among whom $1(1.4 \%)$ had direct hernia, and $1(1.26 \%)$ had indirect hernia having a p-value of 0.933 . In a recent large meta-analysis comparing the two procedures the authors studied the results of ten randomized controlled trials and observed that patients undergoing TEP procedure showed no significant difference between operation time, hospital stay time, recovery period and rate of complications as compared to TAPP. There was no significant association between recurrence rates and postoperative pain scores respectively. They found both the procedures to be equally safe..$^{15,16}$ We propose that surgeons be well prepared in advance for a conversion, and inform the patient about possible conversion of the procedure along with the possible complications. The main limitation of our study was a small sample size and the inclusion of only male patients.

\section{CONCLUSION}

According to the results of our study, $14 \%$ of cases of direct and indirect hernia had to convert from TEP to TAPP procedure, and the main reasons for this conversion were found to be hemorrhage, peritoneal tear and instrument failure.

Copyright@ 06 May, 2019.

\section{REFERENCES}

1. Chung L, Noeeir J, O'Dwyer J. Long-term follow-up of patients with a painless inguinal hernia from a randomized clinical trial. Br J Surg 2011; 98:596-9.

2. Rosenberg J, Bisgaard $T$, Kehlet $H$, Wara $P$, Asmussen T, Juul P, et al. Danish Hernia Database recommendations for the management of inguinal and femoral hernia in adults. Danish Med Bull2011; 58:C4243.

3. Gass M, Banz V, Rosella L, Adamina M, Candinas D, Giiller U. TAPP or TEP? Population-based analysis of prospective data on 4,552 patients undergoing endoscopic inguinal hernia repair. World J Surg 2012; 36:2782-6. 
4. Moreno-EgeaA, Paredes P, Perella J, CampilloSotoA, Baena E, Muii.oz J, et al. Vascular injury by tacks during total extra peritoneal endoscopic inguinal hernioplasty. Surg Laparosc Endosc2010; 20:129-31.

5. O? Reilly AE, Burke, PJ, O? Connell, Ronan P. A meta analysis of surgical morbidity and recurrence after laparoscopic and open repair of primary unilateral inguinalhernia. Ann Surg Treat Res2012; 255:846-53.

6. Choi YY, Kim Z, Hur KY. Learning curve for Total extraperitoneal repair of inguinal hernia. Can $\mathrm{J}$ Surg 2012; 55:336.

7. Jeon $\mathrm{Y}, \mathrm{Kim}$ M, Hur K. Laparoscopic inguinal hernia repair by intraperitonealonlay mesh (IPOM) technique in specific cases as an alternative method. J Minim Invasive Surg2014; 17:30.

8. Simons MP, Aufenacker T, Bay-Nielsen M, Bouillot JL, Campanelli G; Conze J, et al. European Hernia Society guidelines on the treatment of inguinal hernia in adult patients. Hernia 2009; 13:343403.

9. Fitzgibbons RJ, Forse RA. Clinical practice. Groin hernias in adults. TheNEngl JMed2015; 372:75663.

10. Gray $\mathrm{SH}$, HawnMT, Itani KM. Surgical progress in inguinal and ventral incisional hernia repair. Surg Clin NorthAm 2008; 88:17-26
11. Woods B, Neumayer L. Open repair of inguinal hernia: An evidence-based review. Surg Clin North Am 2008; 88:139-55.

12. Sanabria A, Dominguez LC, Valdivieso E, Gomez $G$ Prophylactic antibiotics for mesh inguinal hernioplasty: A meta-analysis. Ann Surg 2007; 245:392-6.

13. Chung L, Norrie J, O’Dwyer PJ. Long-term follow-up of patients with a painless inguinal hernia from a randomized clinical trial. Br J Surg2010; 23:50-55

14. Dulucq JL, Wmtringer $P$, Mahajna A. Occult hernias detected by laparoscopic totally extraperitoneal inguinal hernia repair: A prospective study. Hernia 2011; 15:399-402.

15. Ates M, Dirican A, Ozgor D, Gonultas F, Isik B. Conversion to stoppa procedure in laparoscopic total extra peritoneal inguinal hernia repair. JSLS 2012; 16:250-4.

16. Wei FX, Zhang YC, Han W, Zhang YL, Shao Y, Ni R. Transabdominal preperitoneal (TAPP) versus totally extraperitoneal (TEP) for laparoscopic hernia repair: A Meta-Analysis. Surg Laparosc Endosc Percutan Tech. 2015 Oct; 25(5):375-83.

\begin{tabular}{|c|c|c|c|}
\hline \multicolumn{4}{|c|}{ AUTHORSHIP AND CONTRIBUTION DECLARATION } \\
\hline Sr. \# & Author(s) Full Name & Contribution to the paper & Author(s) Signature \\
\hline 1 & $\begin{array}{l}\text { M. Ghayasuddin } \\
\text { Fareya Usmani }\end{array}$ & $\begin{array}{l}\text { Conceptualization, write up, data } \\
\text { collection, literature review, proof } \\
\text { reading. } \\
\text { Literature review, data collection, } \\
\text { write up, analysis, proof reading. }\end{array}$ & M.chengen \\
\hline 3 & Imran Munir & $\begin{array}{l}\text { Data collection, write up, analysis, } \\
\text { literature review. }\end{array}$ & M.Geypendedin \\
\hline 4 & Mahwash Anjum Shafiq & $\begin{array}{l}\text { Data collection, literature review, } \\
\text { initial write up. }\end{array}$ & \\
\hline 5 & Syed M. Sheraz Raza Naqvi & $\begin{array}{l}\text { Corresponding author, literature } \\
\text { review. }\end{array}$ & \\
\hline
\end{tabular}

